\title{
STANDARD VERSUS HEMODYNAMIC PLUS 19-MM ST JUDE MEDICAL AORTIC VALVES
}

Gennaro Ismeno, MD

Attilio Renzulli, MD, FECTS

Marisa De Feo, MD

Alessandro Della Corte, MD

Francesco Enrico Covino, MD

Maurizio Cotrufo, MD, FECTS
Objective: We reviewed our experience with aortic valve replacement using 19-mm St Jude Medical prostheses (St Jude Medical, Inc, St Paul, Minn) in 119 patients, among which 68 (group A) had a Standard model and 51 (group B) had a Hemodynamic Plus model.

Methods: Comparison between the 2 models included analysis of early and late mortality and all valve-related complications. Postoperative echocardiography was performed to evaluate the hemodynamic performance of both prosthetic models. Laboratory tests were performed to evaluate the amount of red blood cell damage caused by the transprosthetic turbulent flow.

Results: Average body surface area was $1.66 \pm 0.14 \mathrm{~m}^{2}$ in group A and 1.65 $\pm 0.16 \mathrm{~m}^{2}$ in group B $(P=.72)$. There was no statistically significant difference between the 2 groups in terms of preoperative variables (sex, cardiac rhythm, body surface area, preoperative gradients, and New York Heart Association class). Five-year follow-up was $100 \%$ complete. Although group A patients had significantly higher postoperative peak and mean gradients $(P=.0001)$ and a lower effective orifice area $(P=.0001)$, no statistical differences were found in terms of late $(5$-year) survival $(P=.6)$ and postoperative complications $(P=.09)$. Moreover, postoperative left ventricular mass was found to be similar in the 2 groups $(P=.18)$. Hematologic evaluation did not show any significant difference between the 2 groups as to incidence of hemolysis.

Conclusions: Aortic valve replacement with 19-mm aortic prostheses in patients with a body surface area of less than $1.7 \mathrm{~m}^{2}$ allows good results. Although Hemodynamic Plus models have better hemodynamic results, no significant difference was found in terms of clinical results and clinical hemolysis. (J Thorac Cardiovasc Surg 2001;121:723-8)

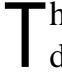
he replacement of the aortic valve with any kind of device implies the acceptance of a residual gradient. ${ }^{1-3}$ The use of an aortic valve prosthesis in the small anulus raises concerns about the harmful effects of residual obstruction to left ventricular outflow. ${ }^{4-7}$ Residual gradient is the driving stimulus for incomplete regression of left ventricular hypertrophy after aortic valve replacement (AVR), and it has been related to higher long-term incidence of cardiac complications and mortality. ${ }^{8-11}$ An indexed orifice area of less than $0.9 \mathrm{~cm}^{2} / \mathrm{m}^{2}$ has been associated with a higher incidence of late complications, including sudden death. ${ }^{3,12}$

From the Department of Cardio-Thoracic and Respiratory Sciences, V. Monaldi Hospital, Second University of Naples, Italy.

Copyright (c) 2001 by The American Association for Thoracic Surgery

0022-5223/2001 $\$ 35.00+0 \quad \mathbf{1 2 / 1 / 1 1 3 0 2 3}$

doi: $10.1067 / \mathrm{mtc} .2001 .113023$
Tilting disc and stented porcine 19-mm valves have both shown poor hemodynamic performance in the aortic position. ${ }^{1,6,11,13}$ On the other hand, bileaflet valves are still the most implanted cardiac valve substitutes in the aortic position. Their excellent durability and low incidence of cardiac-related complications have been widely reported in the medical literature. ${ }^{1,5,14,15}$

Although valve manufacturers have recently improved hemodynamic performances of small aortic prostheses, the implantation of small-valve prostheses in the aortic position is still considered to imply the risk of high residual gradients. $5,6,15,16$

The purpose of this retrospective study is to evaluate and compare the clinical and hemodynamic performance of 19-mm St Jude Medical Hemodynamic Plus (HP) and Standard valves (St Jude Medical, Inc, St Paul, Minn) implanted in the aortic position. 
Table I. Preoperative clinical and echocardiographic data

\begin{tabular}{lccc}
\hline & Group A & Group B & P value \\
\hline No. of patients & 68 & 51 & .5 \\
Age (y) & $65.15 \pm 11.41$ & $63.04 \pm 13.12$ & .36 \\
Sex (M/F) & $20 / 48$ & $20 / 31$ & .81 \\
Preoperative ECG rhythm & 66 SR & 49 SR & .72 \\
Mean BSA, m ${ }^{2}$ (range) & $1.66 \pm 0.14(1.4-1.69)$ & $1.65 \pm 0.16(1.36-1.69)$ & .8 \\
Ascending aortic diameter (mm) & $29.33 \pm 3.5$ & $30 \pm 3.5$ & .5 \\
Max gradient (mm Hg) & $92.11 \pm 23.06$ & $85.14 \pm 22.1$ & .7 \\
Mean gradient (mm Hg) & $54.6 \pm 15.9$ & $51.33 \pm 16.7$ & .8 \\
NYHA class & $2.41 \pm 0.4$ & $2.47 \pm 0.5$ & $.0001^{*}$ \\
Total length of follow-up (mean in mo) & $4739(77.69 \pm 35.04)$ & $1406(28.12 \pm 14.95)$ & \\
\hline
\end{tabular}

$S R$, Sinus rhythm; NYHA, New York Heart Association.

*Statistically significant difference.

Table II. Late echocardiographic data

\begin{tabular}{lccc}
\hline Echocardiographic parameters & Group $A(n=58)$ & Group B $(n=49)$ & P value \\
\hline EF $(\%)$ & $57 \pm 7$ & $61 \pm 7$ & $.004^{*}$ \\
Peak gradient $(\mathrm{mm} \mathrm{Hg})$ & $53.85 \pm 7.16$ & $32.78 \pm 11.72$ & $.0001^{*}$ \\
Mean gradient $(\mathrm{mm} \mathrm{Hg})$ & $34.80 \pm 5.55$ & $19.11 \pm 8.85$ & $.0001^{*}$ \\
Interventricular septum $(\mathrm{mm})$ & $12.2 \pm 3.8$ & $12.6 \pm 1.6$ & .493 \\
Posterior wall $(\mathrm{mm})$ & $7.6 \pm 5.9$ & $12.5 \pm 0.6$ & $.0001^{*}$ \\
Ascending aortic diameter $(\mathrm{mm})$ & $31.0 \pm 4.5$ & $33.8 \pm 8.0$ & $.026^{*}$ \\
EOA $\left(\mathrm{cm}^{2}\right)$ & $1.93 \pm 0.05$ & $2.02 \pm 0.06$ & $.0001^{*}$ \\
IEOA $\left(\mathrm{cm}^{2} / \mathrm{m}^{2}\right)$ & $1.17 \pm 0.04$ & $1.21 \pm 0.05$ & $.0001^{*}$ \\
LVM $(\mathrm{g})$ & $236 \pm 53$ & $216 \pm 46$ & $.04^{*}$ \\
Indexed LVM $\left(\mathrm{g} / \mathrm{m}^{2}\right)$ & $142 \pm 45$ & $131 \pm 38$ & .18 \\
\hline
\end{tabular}

$E F$, Ejection fraction.

*Statistically significant difference.

\section{Materials and methods}

Between January 1983 and December 1997, 119 isolated 19-mm St Jude Medical bileaflet aortic prostheses were implanted at our institution. Patients were distributed into 2 groups according to the prosthesis model: group A included 68 patients with the Standard model, and group B included 51 patients with the HP model. Preoperative clinical and echocardiographic data are reported in Tables I and II, respectively.

Patients. Patient selection was not randomized; the St Jude Medical HP valve has been available in our institution since 1994, and therefore the majority of group A patients were operated on earlier than group B patients. All patients had aortic stenosis; concomitant aortic regurgitation was observed in $10(14.7 \%)$ patients undergoing AVR with the Standard prosthesis and in $11(21.6 \%)$ undergoing AVR with the HP model $(P=.46)$. Patients with associated procedures for coronary artery disease, mitral valve disease, and/or discrete subvalvular aortic stenosis were excluded from the study. Furthermore, to avoid any interference between valve-related hemolysis and red blood cell damage caused by other diseases, patients with more than moderate tricuspid regurgitation; hepatic disease, renal disease, or both; or any kind of hematologic disorder were excluded.

Surgical procedure. After median sternotomy, all patients underwent AVR with a $19-\mathrm{mm}$ mechanical bileaflet prosthe- sis under mild hypothermic cardiopulmonary bypass. Myocardial protection was achieved both with topical hypothermia and with intermittent infusion of cold crystalloid solution (St Thomas' Hospital) directly into the coronary ostia. The procedure was always performed by the same group of surgeons. ${ }^{12}$ Aortic prostheses were implanted in the intra-annular position with nonpledget-supported simple interrupted sutures (Tevdek 2-0; Deknatel, Inc, Fall River, Mass). The hinge area was positioned at $90^{\circ}$ with the interventricular septum. By policy, in our experience an associate patch enlargement of the aortic anulus was considered only in patients with an expected indexed effective orifice area (IEOA) of less than $0.9 \mathrm{~cm}^{2} / \mathrm{m}^{2}$.

Anticoagulation protocol. All patients were treated with oral anticoagulation, which was carried out in all cases with warfarin sodium. Ninety percent of our patients were followed up in our anticoagulation clinic. The others were referred to a satellite institution following the same anticoagulation protocol as our center. Prothrombin activity was kept between $25 \%$ and $35 \%$, which equals an international normalized ratio ranging between 2.0 and 3.5.

Clinical evaluation and definition of complications. Patients operated on in our institution are commonly seen at least yearly in our outpatient clinic where they undergo clinical examination (including blood pressure measurement) and, when indicated, echocardiographic study. Hospital 


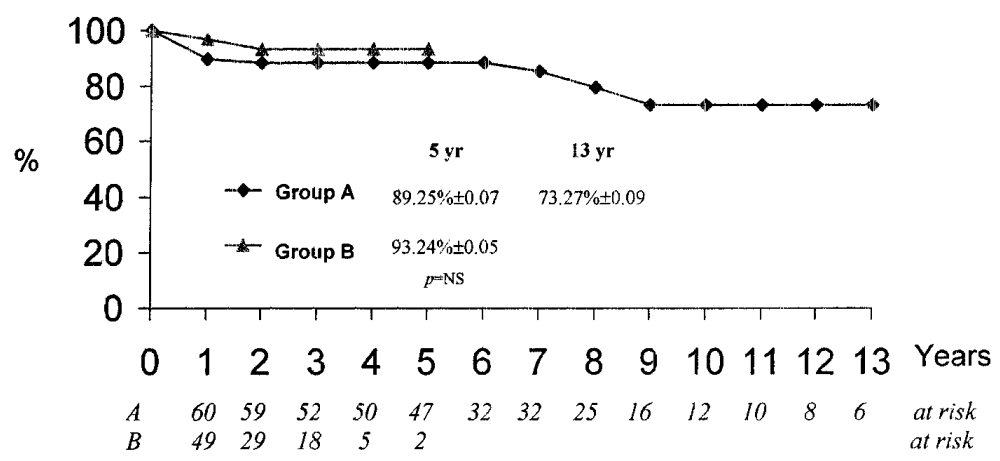

Fig 1. Freedom from treatment failure in the 2 groups of patients with 19-mm St Jude Medical aortic prostheses.

records were checked retrospectively to achieve hospital mortality and morbidity data. No patient was lost to follow-up. Cumulative mortality and morbidity was labeled as "treatment failure" (including hospital mortality, late mortality, and all valve-related complications). ${ }^{17,18}$

Echocardiographic study. All surviving patients underwent M-B mode color Doppler echocardiographic evaluation preoperatively and during the follow-up period. Postoperative echocardiography was performed every 6 months.

The modified Bernoulli equation was used to calculate the instantaneous pressure gradients across the prosthesis. The velocity in the left ventricular outflow tract (LVOT) was considered in gradient calculation by including it in the equation. The mean pressure gradient was calculated by averaging the gradient at 40-ms intervals throughout the velocity complexes. Peak velocity measurements were performed with a pulsed wave Doppler echocardiographic scanner by using the lower angle as soon as possible. The peak velocity was obtained by averaging from 3 measured velocities. Velocities across the prosthesis were recorded a few minutes after the end of the exercise because at this time the peak flow velocity reaches its highest level. The effective orifice area (EOA) of the prosthesis was calculated with the following continuity equation by mean of the simplified peak velocity method:

$$
\mathrm{EOA}=\mathrm{CSA}\left(\mathrm{PkV}_{\mathrm{LVOT}} / \mathrm{PkV}_{\text {jet }}\right)
$$

where CSA is the cross-sectional area of the LVOT, and $\mathrm{PkV}_{\text {LVOT }}$ and $\mathrm{PkV}_{\text {jet }}$ are the maximal velocity in the LVOT and across the valve, respectively. This simplified method has shown a good correlation with that of the original continuity equation. Furthermore, the cross-sectional area of the LVOT was determined by assuming that the sewing ring size was the cross-sectional diameter of the LVOT.

In all cases the IEOA (IEOA = EOA/BSA [body surface area]) and indexed left ventricular mass (LVM) were calculated. BSA was calculated according to the formula of Dubois.

Hematologic evaluation of hemolysis. All patients were informed about the purpose of the study, and all of them gave written consent. A $20-\mathrm{mL}$ blood sample was taken every week for 2 weeks to perform the following measurements: (1) blood hemoglobin (in grams per deciliter); (2) serum lactic dehydrogenase (in units per liter); (3) percent-correlated reticulocyte fraction; (4) serum haptoglobin (in grams per liter); and (5) schistocytes (percent). The data were recorded, and the final results were expressed as the mean value of the 2 blood tests.

The criteria proposed by Skoularigis and colleagues ${ }^{19}$ were used to define hemolysis. Patients were considered as having intravascular hemolysis when serum lactic dehydrogenase (LDH) levels were elevated (>460 U/L, major criteria) and at least 2 of the following minor criteria were observed:

- blood hemoglobin of less than $13.8 \mathrm{~g} / \mathrm{dL}$ for male patients and less than $12.4 \mathrm{~g} / \mathrm{dL}$ for female patients;

- reticulocyte fraction of greater than $2 \%$;

- presence of schistocytes in the blood; and

- serum haptoglobin level of less than $0.5 \mathrm{~g} / \mathrm{L}$.

Statistical analysis. Statistical analysis was performed with the SPSS statistical software package (SPSS, Inc, Chicago, Ill). Categoric values of the 2 groups were compared by using the $\chi^{2}$ or Fisher exact tests, whereas continuous data, reported as means $\pm \mathrm{SD}$, were compared by using the Student $t$ test. Time-related analysis of late complications was calculated as the percentage of events per year. Univariate survival and actuarial analysis were performed with the Kaplan-Meier method and were compared with the log-rank test.

\section{Results}

\section{Clinical results}

Hospital mortality. A total of $8(6.7 \%)$ patients died during their hospital stay; $7(10.3 \%)$ belonged to group $\mathrm{A}$, and $1(2 \%)$ belonged to group B $(P=.153)$. Causes of death were low output syndrome in 6 patients $(5$ patients of group A and 1 patient of group $\mathrm{B} ; P=.08$ ), mediastinitis in 1 patient, and disseminate intravascular coagulopathy in 1 patient.

Late mortality. Four patients died during the followup; $3(4.9 \%)$ of them had a Standard model prosthesis (group A), and $1(2 \%)$ had an HP model prosthesis (group B) $(P=.757)$. Causes of late death were sudden death in 2 patients, both belonging to group $A$ 
Table III. Hematologic results (evaluation of hemolysis)

\begin{tabular}{lccc} 
& Group A & Group B & P value \\
\hline Hemolysis & $14 / 58(24.14 \%)$ & $13 / 49(26.53 \%)$ & .993 \\
Serum haptoglobin (g/L) & $1.8 \pm 0.2$ & $2.2 \pm 0.5$ & $.0001^{*}$ \\
Reticulocytes (\%) & $0.5 \pm 0.5$ & $1.2 \pm 0.21$ & $.0001^{*}$ \\
Schistocytes (\%) & $1.1 \pm 0.4$ & $0.9 \pm 0.3$ & $.005^{*}$ \\
LDH (U/L) & $610 \pm 62$ & $580 \pm 58$ & $.011^{*}$ \\
Blood hemoglobin (g/dL) & $13.4 \pm 1.4$ & $13.7 \pm 1.6$ & .299
\end{tabular}

*Statistically significant difference.

(transprosthetic mean gradients at the last echocardiographic control were 25 and $35 \mathrm{~mm} \mathrm{Hg}$, respectively); cancer in 1 patient; and road accident in 1 patient. Fiveyear actuarial survival did not show any significant difference between the 2 groups (group A, $93.4 \% \pm 4 \%$; group $\mathrm{B}, 96.15 \% \pm 37 \%, P=.6$; 13 -year actuarial survival in group A was $90.89 \% \pm 6 \%$ ).

Clinical status. Mean New York Heart Association class at latest follow-up was $1.2 \pm 0.7$ in group A versus $1.1 \pm 0.6$ in group B $(P=.14)$. There was no statistical difference between the 2 groups in terms of peripheral blood pressure (group A: mean systolic pressure of $145 \pm 15 \mathrm{~mm} \mathrm{Hg}$ and mean diastolic pressure of $80 \pm 10 \mathrm{~mm} \mathrm{Hg}$; group B: mean systolic pressure of $140 \pm 20 \mathrm{~mm} \mathrm{Hg}$ and mean diastolic pressure of $75 \pm 10 \mathrm{~mm} \mathrm{Hg}$ ) at follow-up. Moreover, there was no statistical difference between the 2 groups in terms of requirement of cardiovascular drugs (group A, 40/61 [65.6\%] patients; group B, 32/50 [64\%] patients).

Embolism. There was no statistical difference between rates of freedom from embolism in the 2 groups at 5 years of follow-up. No case of embolism was observed in any group of patients (100\% event free) within the first 5 years of follow-up. Two patients of group A had a neurologic complication caused by embolism after the fifth postoperative year, resulting in a 13 -year event-free rate of $89.41 \% \pm 7 \%$.

Hemorrhage. No case of hemorrhage was observed in patients with HP prostheses (100\% event free). One patient with the Standard 19-mm prosthesis had gastric bleeding that required blood transfusion (13-year freedom from hemorrhage in group A, $98.25 \% \pm 2 \%$ ). No statistical difference was found between the 2 groups at 5 years of follow-up.

Endocarditis, thrombosis, and reoperation. No cases of endocarditis, prosthetic thrombosis, and reoperation have been reported during the follow-up.

Treatment failure. Cumulative analysis of events, including early and late mortality and all valve-related complications, did not show any statistically significant difference at 5 years of follow-up (group A, $89.25 \% \pm$
7\%; group B, 93.24\% $\pm 5 \% ; P=.09$ ). Thirteen-year freedom from treatment failure in group A was $73.27 \%$ $\pm 9 \%$ (Fig 1).

Echocardiographic results. Late echocardiographic results are reported in Table II. Significantly lower mean $(19.11 \pm 8.85 \mathrm{~mm} \mathrm{Hg})$ and peak gradients $(32.78 \pm 11.72 \mathrm{~mm} \mathrm{Hg})$ were observed in patients with the St Jude Medical HP prosthesis versus the Standard prosthesis (mean gradient, $34.80 \pm$ $5.55 \mathrm{~mm} \mathrm{Hg}$; peak gradient, $53.85 \pm 7.16 \mathrm{~mm} \mathrm{Hg}$; $P=.001)$. EOA was statistically higher $(P=.0001)$ in patients with HP heart valve prostheses $(2.02 \pm$ 0.06) versus Standard St Jude Medical prostheses $(1.93 \pm 0.05)$. Similar data were observed in the analysis of the IEOA (Table II). No statistical difference $(P=.18)$ was observed between the mean LVM of the HP prosthesis group $(131 \pm 38 \mathrm{~g})$ and that of the Standard prosthesis group $(142 \pm 45 \mathrm{~g})$.

Hematologic results. No pathologic levels of serum haptoglobin, reticulocytes, and schistocytes were observed in both groups. Haptoglobin levels and percentcorrected reticulocyte fractions were statistically lower in patients with the Standard prosthesis (Table III). The mean count of schistocytes was statistically lower $(P=$ $.005)$ in patients with the HP prosthesis (Table III).

The mean LDH value was high ( $>460 \mathrm{U} / \mathrm{L})$ in both groups; the mean $\mathrm{LDH}$ value of $580 \pm 58 \mathrm{U} / \mathrm{L}$, which was observed in group B patients, was statistically lower than $610 \pm 62 \mathrm{U} / \mathrm{L}$, which was observed in patients with Standard prostheses $(P=.011$, Table III).

Low levels of blood hemoglobin $(13.7 \pm 1.6 \mathrm{~g} / \mathrm{dL}$ in group B patients and $13.4 \pm 1.4 \mathrm{~g} / \mathrm{dL}$ in group $\mathrm{A}$ patients) were found ( $P=$ not significant).

According to the criteria ${ }^{19}$ previously reported, 14 (24.14\%) of 58 group A patients and 13 (26.5\%) of 49 group B patients showed some degree of intravascular hemolysis $(P=$ not significant, Table III).

\section{Discussion}

The objective of AVR is to restore valvular function with the lowest transprosthetic gradient. To improve hemodynamic performances of aortic valve prostheses, 
new models of bileaflet valves have been devised, reducing the suture cuff. ${ }^{6,15}$ At the same time, stentless porcine valves have been commercialized to minimize transprosthetic gradients. ${ }^{9,10,20}$ In patients with stentless valves, minimal transprosthetic gradients and almost complete LVM regression have been reported. ${ }^{9,20}$

In the early decades of cardiac operations, hemodynamic evaluation of mechanical aortic prostheses was almost impossible. ${ }^{21-23}$ Surgeons used to evaluate transprosthetic gradients intraoperatively.

Doppler ultrasonography-measured pressure gradients across different types of bioprosthetic and mechanical valves are reported to correlate well with catheter-derived gradients. ${ }^{24-26}$ Although bileaflet valves have a central flow and should allow an accurate measurement of gradients, the presence of pressure recovery and high localized pressure gradients might cause some overestimation compared with the catheter pressure gradient, as found by Baumgartner and associates $^{26}$ in St Jude Medical valves. The results of a proper match between patients and prostheses can be evaluated by means of echocardiography through ventricular mass regression. $2,9,12,13$

Nowadays, assessment of bileaflet valve performances must include clinical and echocardiographic studies. In addition, high gradients and flow turbulence have been associated with significant hemolysis. ${ }^{19,27,28}$

Many studies, in which the performances of different aortic valve prostheses were evaluated, have emphasized that successful AVR should achieve an indexed valve area greater than $0.9 \mathrm{~cm}^{2} / \mathrm{m}^{2}$ to minimize prosthetic gradients and obtain postoperative LVM regression. . $^{3,8,11,12}$

Therefore, there are some controversies about the long-term effect of residual transprosthetic gradient in patients with a small aortic anulus and an expected IEOA greater than $0.9 \mathrm{~cm}^{2} / \mathrm{m}^{2}$. Furthermore, the advantages and the intermediate-term results of new and improved orifice models are still to be fully assessed.

Transprosthetic gradients are correlated to cardiac output and BSA. The BSA of Mediterranean patients older than 60 years referred for AVR averages $1.66 \mathrm{~m}^{2}$. In our study, a critical value of IEOA of less than 0.9 was not observed in any patient.

This study confirms that as long as the EOA of bileaflet aortic valve implanted in adult patients with normal body size is greater than 0.9 to $1 \mathrm{~cm}^{2} / \mathrm{m}^{2}$, hemodynamic performances are good, and long-term clinical results are excellent. Hachida and colleagues, ${ }^{29}$ although studying a group of patients with smaller BSAs than ours and with pure aortic stenosis alone, reported postoperative results that are consistent with our findings as far as peak and mean gradients and postoperative indexed LVM are concerned, particularly in patients with HP models. However, we could not explain the discrepancy between that study and ours in the postoperative EAO of both Standard and HP valves. In no study have HP valves, despite their better hemodynamic results, been shown to significantly improve clinical results. However, a $91 \%$ survival at 13 years in patients with Standard model prostheses and a mean age of 65 years is already a good result when compared with other recent series. ${ }^{30,31}$

This study analyses our 16-year experience with 19 mm Standard and HP St Jude Medical aortic prostheses retrospectively. A retrospective analysis of such a wide time period may imply limitations related to changes in diagnostic, operative, or both types of technologies and techniques. Length of follow-up is different between the 2 groups because HP models have been available in our institution since 1994. However, the longer followup belongs to the model that has worse hemodynamic performances. Moreover, time-related analysis of events was truncated at 5 years for both groups to achieve comparable data. Although in our study no significant difference between the 2 groups of patients was found in terms of late survival, it must be pointed out that most of the patients from group B have not yet reached 4 years of follow-up. No LVM regression analysis was performed because such data have been available in our institution only in recent years. Another limitation to our study can be represented by the mean age of patients because a lower physical activity is expected in patients older than 60 years.

It must be pointed that because bileaflet valves in the aortic position usually carry low degrees of hemolysis, ${ }^{19}$ differences between similar models can be difficult to ascertain with the common hematologic tests. ${ }^{28}$ The criteria of Skoularigis and colleagues ${ }^{19}$ have been proven to be effective in evaluating the amount of intravascular hemolysis and to perform comparisons between different models of mechanical valves. Nevertheless, according to such criteria, no significant difference between the HP and Standard series was found as to late postoperative intravascular hemolysis, although mean LDH serum levels and schistocyte count were significantly lower in group B. It can be stated that subclinical blood cell damage could be significantly higher in patients with standard 19-mm aortic prostheses compared with those receiving HP prostheses.

In conclusion, our study confirms that $19-\mathrm{mm}$ bileaflet aortic valves allow good long-term clinical results and acceptable hemodynamic results in patients with a BSA of less than $1.7 \mathrm{~m}^{2}$. Improved orifice models allow lower gradients, but such data do not affect clinical results and the amount of red blood cell damage. 
Received for publication July 18, 2000; revisions requested Aug 22, 2000; revisions received Nov 10, 2000; accepted for publication Nov 14, 2000.

Address for reprints: Attilio Renzulli, MD, FECTS, Via Aquila 144, 80143, Napoli, Italy (E-mail: arenzul@tin.it renzulliattilio@usanet).

\section{REFERENCES}

1. Yoganathan AP, Chaux A, Gray RJ, Woo YR, DeRobertis M, Williams FP, et al. Bileaflet, tilting disc and porcine aortic valve substitutes: in-vitro hydrodynamic characteristics. J Am Coll Cardiol 1984;3:313-20.

2. Dumesnil JG, Yoganathan AP. Valve prosthesis hemodynamic and the problem of high transprosthetic pressure gradient. Eur $\mathrm{J}$ Cardiothorac Surg 1992;6(Suppl 1):S34-8.

3. Rashtian MY, Stevenson DM, Allen DT, Yoganathan AP, Harrison EC, Edmiston WA, et al. Flow characteristics of four commonly used mechanical heart valves. Am J Cardiol 1986;58:743-52.

4. He GW, Grunkemeier GL, Gately HL, Furnary AP, Starr A. Up to thirty-year survival after aortic valve replacement in the small aortic root. Ann Thorac Surg 1995;59:1056-62.

5. Aoyagi S, Arinaga K, Fukunaga S, Kawano H, Kawara T. Aortic valve replacement with a small mechanical valve prosthesis-a clinical and echocardiographic study of a St Jude Medical valve prosthesis. Jpn Circ J 1998;62:244-8.

6. Noera G, Pensa PM, Lamarra M, Mascagni R, Cremonesi A, Balestra G. Hemodynamic evaluation of the CarboMedics R, St Jude Medical HP and Sorin Bicarbon valve in patients with small aortic annulus. Eur J Cardiothorac Surg 1997;11:473-6.

7. Gonzalez-Juanatey JR, Fernandez MV, Sampedro FG, GasrciaAcuna JM, Garcia-Bengocchea JB, Cendom AA, et al. Hemodynamic performance of aortic pericardial bioprostheses and bileaflet prostheses at rest and during excercise: implications for the surgical management of patients with small aortic roots. Heart 1999;82:149-55.

8. Bech-Hanssen O, Caiadahl K, Wall B, Myken P, Larsson S, Wallentin I. Influence of aortic valve replacement, prosthetic type, and size on functional outcome and ventricular mass in patients with aortic stenosis. J Thorac Cardiovasc Surg 1999;118:57-65.

9. Thomas HL, O'Brien MF, Almeida AA, Tesar PJ, Davison MB, Burstow DJ. Heamodynamic and left ventricular mass regression: a comparison of the stentless, stented and mechanical aortic valve replacement. Eur J Cardiothorac Surg 1998;13:572-5.

10. Jin XY, Pillai R, Westaby S. Medium-term determinants of left ventricular mass index after stentless aortic valve replacement. Ann Thorac Surg 1999;67:411-6.

11. Jin XY, Zhang ZM, Gibson DG, Yacoub MH, Pepper JR. Effects of valve substitute on changes in left ventricular function and hypertrophy after aortic valve replacement. Ann Thorac Surg 1996;62:683-90.

12. Rahimtoola SH. The problem of valve prosthesis-patient mismatch. Circulation 1978;58:20-4.

13. Pibarot P, Honos GN, Durand LG, Dumesnil JG. The effect of prosthetic-patient mismatch on aortic bioprosthetic valve hemodynamic performance. Can J Cardiol 1996;12,4:379-87.

14. D'Alessandro LC, Pucci A, Angelica G, Mamone P, D'Alessandro C, Pisani P, et al. Sixteen-year follow-up of the St Jude Medical prosthesis: evaluation of the indexed prosthesis area in short-and long-term results. Ann Thorac Cardiovasc Surg 1995;5:271-8.
15. Fisher J. Comparative study of the hydrodynamic function of the size $19 \mathrm{~mm}$ and $21 \mathrm{~mm}$ St. Jude Medical Hemodynamic Plus bileaflet heart valves. J Heart Valve Dis 1994;3:75-80.

16. Katz JM, Sade RM, Crawford FA, Crumbley AJ, Stroud MR. The risk of small St. Jude aortic valve prostheses. Ann Thorac Surg 1994;57:1114-9.

17. Edmunds LH Jr, Clark RF, Cohn LH, Grunkemeier GL, Miller DC, Weisel RD. Guidelines for reporting morbidity and mortality after cardiac valvular operations. The American Association for Thoracic Surgery, Ad Hoc Liason Committee for Standardising Definitions of Prosthetic Heart Valve Morbidity. Ann Thorac Surg 1996;62:932-5.

18. de Luca L, Vitale N, Giannolo B, Cafarella G, Piazza L, Cotrufo M. Mid-term follow-up after heart valve replacement with CarboMedics bileaflet prostheses. J Thorac Cardiovasc Surg 1993;106:1158-65.

19. Skoularigis J, Essop MR, Skudicky D, Middlemost SJ, Sareli P. Frequency and severity of intravascular hemolysis after left-sided cardiac valve replacement with Medtronic Hall and St Jude Medical prostheses, and influence of prosthetic type, position, size and number. Am J Cardiol 1993;71:587-91.

20. Del Rizzo DF, Goldman BS, Christakis GT, David TE. Hemodynamic benefits of the Toronto Stentless Valve. J Thorac Cardiovasc Surg 1996;112:1431-45.

21. Lee SJK, Haraphongse M, Callaghan JC, Rossal RE, Fraser RS. Hemodynamic changes following correction of severe aortic stenosis using the Cutter-Smeloff prosthesis. Circulation 1970;42:719-25.

22. Pluth JR, Braodbent JC, Barnhorst DA, Danielson GK. Aortic and mitral valve replacement with cloth-covered BraunwaldCutter prosthesis. Ann Thorac Surg 1975;20:239-44.

23. Björk VO, Holmgren A, Olin C, Orenfors CO. Clinical and haemodynamic results of aortic valve prosthesis. Scand J Thorac Cardiovasc Surg 1971;5:177-81.

24. Reisner SA, Meltzer RS. Normal values of prosthetic valve Doppler echocardiographic parameters: a review. J Am Soc Echocardiogr 1988;1:201-10.

25. Chambers J. Echocardiography and the small aortic root. J Heart Valve Dis 1996;5(Suppl III):S264-8.

26. Baumgartner H, Khan S, De Robertis M, Czer L, Maurer G. Effect of prosthetic valve design on the Doppler-catheter gradient correlation: an in-vitro study of normal St Jude, Medtronic-Hall, Starr-Edwards and Hancock valves. J Am Coll Cardiol 1992;19:324-32

27. Lund O. Late chronic hemolysis after valve replacement for aortic stenosis: relation to residual hypertrophy and impaired left ventricular function. Angiology 1990;41:836-47.

28. Ismeno G, Renzulli A, Carozza A, De Feo M, Iannuzzi M, Santè $\mathrm{P}$, et al. Intravascular hemolysis after mitral and aortic valve replacement with different types of mechanical prostheses. Int J Cardiol 1999;69:179-83.

29. Hachida U, Koyanagi H, Nonoyama M, Bonkohara Y, Saitou S, Nakamura K. Serial hemodynamic study after aortic valve replacement in patients with narrow aortic roots. J Heart Valve Dis 1998;7:81-5.

30. Cohen G, David TE, Ivanov J, Armstrong S, Feindel CM. The impact of age, coronary artery disease, and cardiac comorbidity on late survival after bioprosthetic aortic valve replacement. J Thorac Cardiovasc Surg 1999;117:273-84.

31. He GW, Grunkemeier GL, Starr A. Aortic valve replacement in elderly patients: influence of concomitant coronary grafting on late survival. Ann Thorac Surg 1996;61:1746-51. 